\title{
Correlates of sleep disturbance in symptomatic youth
}

Dominique Bonneville ${ }^{1}$, Jacqueline Stowkowy ${ }^{1}$, Kali Brummitt ${ }^{1}$, Benjamin I. Goldstein ${ }^{2,3}$, Glenda MacQueen ${ }^{1}$, Jean Addington ${ }^{1}$

${ }^{1}$ Hotchkiss Brain Institute, Department of Psychiatry, University of Calgary, Calgary, AB Canada

${ }^{2}$ Centre for Youth Bipolar Disorder, Sunnybrook Health Sciences Centre, Toronto, ON, Canada ${ }^{3}$ Departments of Psychiatry and Pharmacology, Faculty of Medicine, University of Toronto, ON Canada

\section{Abstract}

Sleep disturbances are common across many mental health disorders, with evidence suggesting a bidirectional relationship. Furthermore, there is evidence of a significant association between sleep disruption and worse symptomology in disorders such as anxiety, depression, and psychosis. Additionally, research has demonstrated the negative impact of stress and stressful life events on psychiatric illness progression. However, little is known about possible links between sleep disturbance and these psychiatric symptoms, ongoing stress, stressful life events and functioning, especially in adolescence and early adulthood, a time when many mental illnesses first begin. Exploring these relationships may allow for a better understanding of the role sleep disruption plays in the early stages of illness. Objective: The objective of this study is to investigate potential correlates of sleep disturbance in youth experiencing subthreshold psychiatric symptoms. This study included 160 youth, ages 12 to 25 who were help seeking due to experiencing distress, mild symptoms of anxiety or depression, and/or attenuated syndromes such as clinical high risk for psychosis. Youth meeting criteria for full psychiatric diagnoses were excluded. The Pittsburgh Sleep Quality Index was used to assess sleep disruption. A range of clinical symptoms were assessed using the Scale for Assessment of Psychosis-Risk Symptoms, The Calgary Depression Scale for Schizophrenia and the Generalized Anxiety Disorder 7-Item Scale. Current stress was assessed with the Daily Stress Inventory, and the K10 Distress Scale. Past history of major stress was measured with a Childhood Trauma and Abuse Scale, and the Life Events scale. Fourthly, functioning was assessed with the Global Functioning Scale: Social and Role. Global sleep quality was most highly realted to subthreshold symptoms of anxiety $(r=0.425, p<0.01)$, depression $(r=0.420$, $p<0.01)$, and disorganized symptoms of psychosis $(r=0.232, p<0.05)$. Additionally, global sleep was related to total distress $(r=0.356, p<0.01)$, daily stress $(r=0.268, p<0.01)$, and stressful life events $(r=0.291, p<0.01)$. Social functioning did not reveal any significant correlations with sleep, however, role functioning $(r=0.170, p<0.05)$ did exhibit a relationship with global sleep quality. Finally, bullying $(r=0.196, p<0.05)$ and trauma $(r=0.266, p<0.01)$ were significantly correlated with global sleep quality scores. This study provides early evidence for the interaction that sleep disruption may have with stress, trauma, bullying, and subthreshold psychiatric symptomology in youth. Further research is required to increase the understanding of these relationships early on in youth psychiatric illness progression.

\section{Key words}

serious mental illness, depression, anxiety, psychosis, sleep, youth

Cite as: Bonneville D, Stowkowy J, Brummitt K, Goldstein BI, MacQueen G, Addington J. 2019. Correlates of sleep disturbance in symptomatic youth. Alberta Academic Review, Vol 2 (3) 12, CASCH Special Issue (not peer-reviewed), DOI: 10.29173/aar103. 